\title{
Postoperative negative pressure pulmonary edema following repetitive laryngospasm even after reversal of neuromuscular blockade by sugammadex
} -a case report-

\author{
Ji Hyeon Lee, Jae Ho Lee, Min Hyun Lee, Hyun Oh Cho, and Soon Eun Park \\ Department of Anesthesiology and Pain Medicine, Ulsan University Hospital, University of Ulsan College of \\ Medicine, Ulsan, Korea
}

Laryngospasm, an occlusion of the glottis, can occur at any time during anesthesia, and is associated with serious perioperative complications such as hypoxia, hypercabia, aspiration, bronchospasm, arrhythmia, prolonged recovery, cardiac collapse, and eventually catastrophic death. Importantly, postoperative negative pressure pulmonary edema (NPPE) is a rare, but well described life-threatening complication related to acute and chronic upper airway obstruction. Sugammadex well known for affirmatively reducing the postoperative pulmonary complications associated with residual neuromuscular blockade may have an indirect role in triggering the negative intrathoracic pressure by raising a rapid and efficacious respiratory muscle strength in acute upper airway obstruction. Herein, we report a case of postoperative NPPE following repetitive laryngospasm even after reversal of rocuronium-induced neuromuscular blockade using sugammadex.

Key Words: Laryngismus, Negative pressure pulmonary edema, Rocuronium, Sugammadex.

Laryngospasm, an occlusion of the glottis, is a commonly encountered complication during anesthesia with an overall incidence of 8.7 per 1,000 patients [1]. Although reversible if recognized and managed appropriately, it may be associated

Corresponding author: Ji Hyeon Lee, M.D.

Department of Anesthesiology and Pain Medicine, Ulsan University Hospital, University of Ulsan College of Medicine, 877, Bangeojinsunhwando-ro, Dong-gu, Ulsan 44033, Korea

Tel: 82-52-250-7248, Fax: 82-52-250-7249

Email: jhleesky@naver.com

ORCID: http://orcid.org/0000-0002-8229-3892

Received: July 26, 2016.

Revised: September 27, 2016.

Accepted: October 12, 2016.

Korean J Anesthesiol 2017 February 70(1): 95-99

https://doi.org/10.4097/kjae.2017.70.1.95 with catastrophic consequences owing to rapid occurrence of hypoxia. Not only this, negative pressure pulmonary edema (NPPE) can distress the patient until the postoperative period is over. NPPE has been known to occur as a consequence of laryngospasm in more than $50 \%$ of the patients [2]. Management of NPPE is usually diverse with therapeutic strategies ranging from effective airway management with oxygen and diuretics to mechanical ventilator support in the intensive care unit.

In the present report, we describe a case of postoperative NPPE following repetitive laryngospasm, in a 17-year-old woman, even after reversal of neuromuscular blockade with sugammadex.

\section{Case Report}

A 17-year-old woman (height $150.5 \mathrm{~cm}$, weight $49.6 \mathrm{~kg}$ ) was scheduled for a lateral neck node dissection. She had a history

(c) This is an open-access article distributed under the terms of the Creative Commons Attribution Non-Commercial License (http://creativecommons.org/ licenses/by-nc/4.0/), which permits unrestricted non-commercial use, distribution, and reproduction in any medium, provided the original work is properly cited. 
of papillary thyroid cancer that had been surgically removed a year ago. She did not have any other medical disease and had a good cardiorespiratory functional capacity. At the time of thyroidectomy, the patient had been anesthetized using a bolus of propofol (100 mg), lidocaine (60 $\mathrm{mg}$ ) and rocuronium (50 mg) administered intravenously followed by a maintenance dose of rocuronium (15 mg) given intermittently and continuous administration of remifentanil $(0.05 \mu \mathrm{g} / \mathrm{kg} / \mathrm{min})$. Endotracheal intubation had been performed atraumatically, and the patient was maintained under general anesthesia using desflurane and nitrous oxide. After 3 hours of surgery, the effect of anesthesia were reversed using intravenous pyridostigmine $(15 \mathrm{mg})$ and glycopyrrolate $(0.4 \mathrm{mg})$ intravenously, following which she regained full consciousness, spontaneous breathing, and peripheral motor power. After extubation of the endotracheal tube, she suddenly complained of breathlessness despite a $100 \%$ peripheral oxygen saturation. For this reason, positive pressure of approximately $10 \mathrm{cmH}_{2} \mathrm{O}$ was applied immediately to her airways and the mandible was elevated anteriorly which was followed by administering a bolus of sugammadex $2 \mathrm{mg} / \mathrm{kg}$ in order to exclude the another potential for acute airway obstruction. Her condition gradually returned to normal with sufficient spontaneous ventilation and she recovered fully in the post-anesthetic care unit without developing any further complications.

On a follow-up ultrasound examination, enlarged multiple lymph node was noted. Consequently, she was admitted again for a lateral neck node dissection. The weight of the patient had not changed $(50.6 \mathrm{~kg}$ ) and the laboratory tests including the thyroid function test were within normal ranges. Preoperative electrocardiogram (ECG) was normal sinus rhythm and chest $\mathrm{X}$-ray revealed no active lesion in both lungs. After monitoring ECG, noninvasive blood pressure and pulse oximetry, anesthesia was induced by a bolus of lidocaine (40 mg), propofol (100 mg) and rocuronium (40 mg) administered intravenously. General anesthesia was maintained by desflurane (4-5\%) and nitrous oxide, followed by continuous intravenous infusion of remifentanil $(0.05-0.07 \mu \mathrm{g} / \mathrm{kg} / \mathrm{min})$. In addition, invasive arterial line was inserted and bispectral index system (BIS Quatro sensor, Aspect Medical systems, Norwood, MA, USA) was monitored. For maintenance of neuromuscular relaxation, additional rocuronium was administered at the rate of $10 \mathrm{mg}$ per hour till 90 minutes before the end of surgery. In toto, a dose of $70 \mathrm{mg}$ of rocuronium was administered to the patient during the entire duration of the surgery. A total of 2,700 $\mathrm{ml}$ of fluids in the form of crystalloids and colloids were replenished during the surgery, while estimated blood loss and urine output of the patient were $500 \mathrm{ml}$ and $120 \mathrm{ml}$, respectively. At the end of surgery, which lasted for about 250 minutes, inhalation of desflurane, nitrous oxide and intravenous infusion of remifentanil were discontinued. Seven minutes after the discontinuation of all anesthetic agents, the patient regained consciousness and adequate spontaneous respiration (tidal volume $=400 \mathrm{ml}$ ). After confirming adequate recovery of neuromuscular strength i.e. hand grip, head lift and straight-leg raising, $100 \mathrm{mg}$ of sugammadex at a dose of $2 \mathrm{mg} / \mathrm{kg}$ was administered intravenously instead of pyridostigmine and glycopyrrolate for rapidly reversing a rocuronium induced neuromuscular blockade. However, immediately after the extubation of endotracheal tube, she suddenly stopped breathing and began to gasp. Instantly, oxygen saturation dropped to $70 \%$ and she began to feel drowsy. Positive pressure ventilation was applied shortly by fitting a venturi mask on the airway that helped her regain self-respiration and $100 \%$ oxygen saturation. But during continued assisted mask ventilation, she expelled copious pink frothy sputum and oxygen saturation dropped again to $90 \%$. Coarse inspiratory rhonchi could be clearly heard on bilateral chest auscultation. Though spontaneous breathing returned to normal, she felt a difficulty in deep breathing. Arterial blood gas analysis during oxygen administration $(10 \mathrm{~L} / \mathrm{min})$ revealed a $\mathrm{pH}$ of $7.36, \mathrm{PaCO}_{2}$ of $39 \mathrm{mmHg}, \mathrm{PaO}_{2}$ of $112 \mathrm{mmHg}$, a bicarbonate level of $22 \mathrm{mmol} / \mathrm{L}$ and arterial oxygen saturation of 98\%. She was transferred to the post anesthetic care unit (PACU), where a portable chest radiogram was obtained, which revealed ill-defined heterogeneous but confluent perihilar opacities in both lungs (Fig. 1). Except for this, other vital parameters such as ECG recorded (sinus rhythm) and blood pressure (120/80 $\mathrm{mmHg}$ ) were normal. But she vomited pink frothy sputum once more with oxygen saturation dropping to $95 \%$ in PACU. Since then, her condition was manageable and she showed stable vital signs despite persistent dyspnea. She was transferred to the general ward and her condition was monitored in consultation with

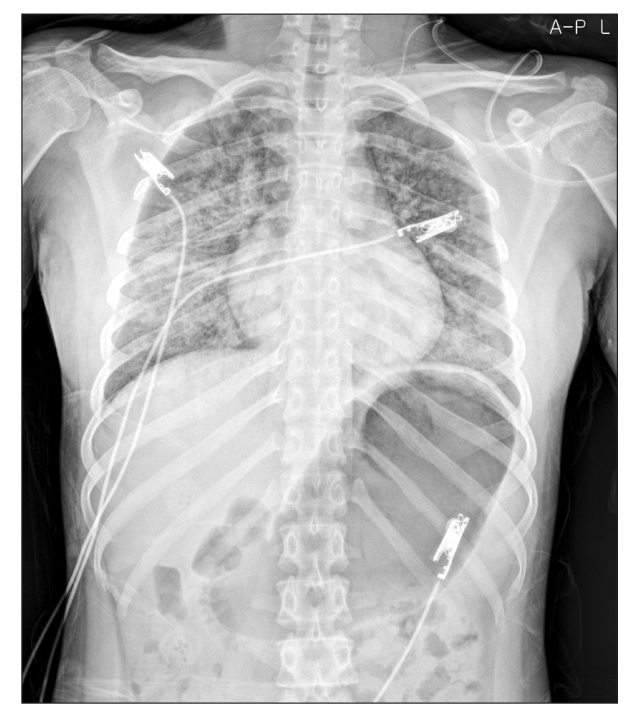

Fig. 1. Portable chest AP radiograph taken in the post anesthetic care unit reveals severe ill-defined heterogeneous confluent perihilar opacities in both lungs. 


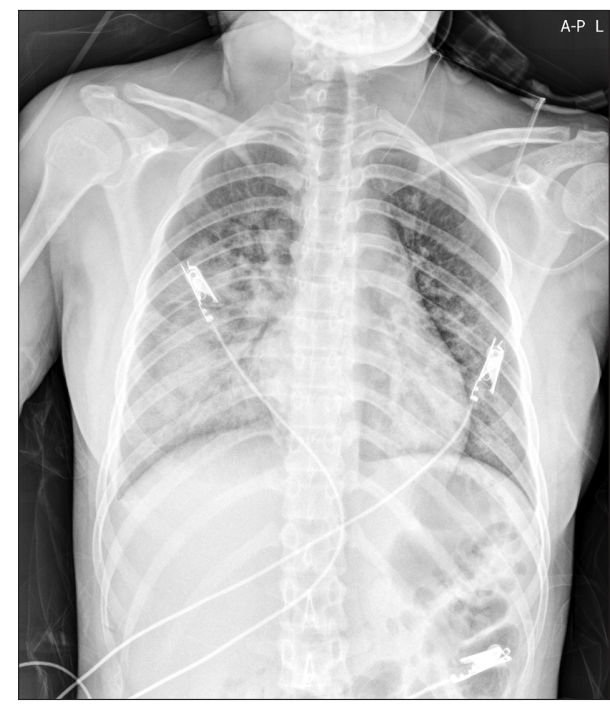

Fig. 2. Portable chest AP radiogram obtained at day 1 of postoperative care shows ill-defined heterogeneous confluent opacities resolving from the upper lung.

the surgeon.

In the general ward, the patient was administered oxygen at the rate of $10 \mathrm{~L} / \mathrm{min}$ with a venturi mask and the peripheral capillary oxygen saturation $\left(\mathrm{SpO}_{2}\right)$ ranged from $91 \%$ to $94 \%$. Her condition became even more manageable with the resolution of dyspnea, 1 day following surgery. Furthermore, the ill-defined heterogeneous confluent opacities began to resolve from the upper lung (Fig. 2) after a day of postoperative hospitalization and only mild ill-defined haziness remained in the right midlower lung (Fig. 3) at day 2 of postoperative care. Six days after the surgery her condition improved significantly with her chest radiogram showing complete remission of opacities and she was eventually discharged from the hospital.

\section{Discussion}

Laryngospasm, which is a prolonged form of vocal cord adduction, can occur at any time during light anesthesia, such as inhalation induction, tracheal intubation, and extubation. It has been observed that laryngeal stimulation as irritating inhalation agents, excessive secretions, or stimulation of visceral nerve endings in the pelvis, abdomen, and thorax may also increase the risk of laryngospasm [3]. In addition to these, factors such as male sex, young age, bronchial asthma, respiratory tract infection, use of vagolytic drug, surgery and manipulation within the pharynx and larynx also precipitate laryngospasm in patients. Above all, manipulation of the airways such as laryngoscopy with tracheal intubation has been identified as the main risk factor for anesthesia induced laryngospasm [4]. Afferent stimulation from multiple receptors like mechanoreceptors, che-

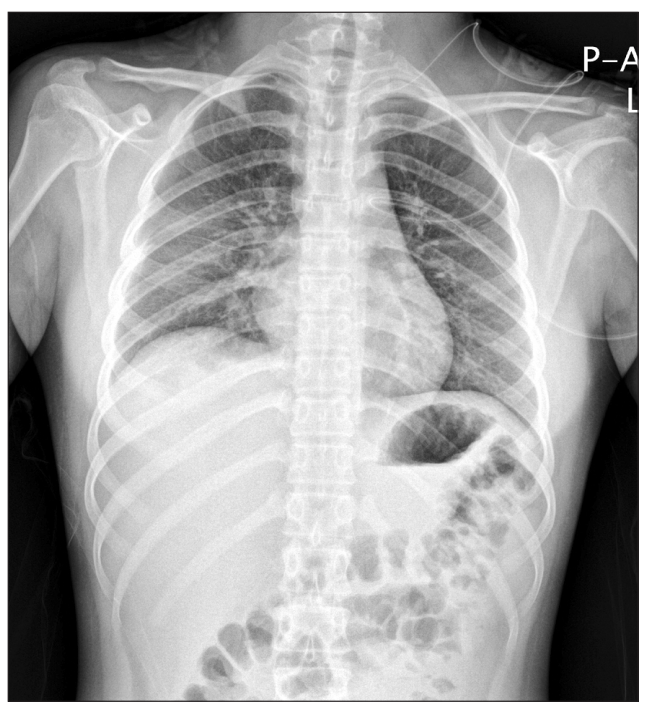

Fig. 3. Chest PA radiogram obtained at day 2 of postoperative care shows only mild ill-defined haziness in right mid-lower lung.

moreceptors, and thermal receptors induce laryngeal closure, triggering exaggeration of the glottis closure reflex and inducing laryngospasm. If not managed promptly by administering continuous positive airway pressure with $100 \%$ oxygen or succinylcholine intravenously, the obstruction of vocal cord leads to hypoxia, hypercarbia, aspiration, bronchospasm, arrhythmia, prolonged recovery, cardiac collapse, and eventually catastrophic death $[3,4]$. Besides, by inducing extremely negative intrapleural pressure due to airway obstruction, laryngospasm may induce fatal pulmonary edema [5]. Consequently, laryngospasm requires careful monitoring by the the anesthesiologist and immediate treatment.

NPPE is rare, and well described postoperative complication, but that can be extremely life threatening for the patient. Also known as post obstructive pulmonary edema (POPE), NPPE, is associated with various pulmonary obstructive conditions and has been classified into two types. While Type I POPE occurs after aggressive inspiration against an acute upper airway obstruction such as during laryngospasm, foreign body aspiration, endotracheal intubation, type II POPE often develops after the relief of chronic airway obstruction such as in the case of upper airway tumor, obstructive sleep apnea [6]. In an acute airway obstruction, the patient makes an extreme inspiratory effort against a blocked airway which generates a highly negative intrathoracic pressures ranging from -40 to $-140 \mathrm{cmH}_{2} \mathrm{O}$ [7]. This event causes hemodynamic changes such as increase in venous return and pulmonary circulation, eventually leading to a decrease in left ventricular compliance and cardiac output [8]. Due to highly negative intrapleural pressure, expanded volume of pulmonary capillaries induces accumulation of fluid into the 
pulmonary interstitial space by elevating hydrostatic pressure. This pulmonary capillary leak results in alveolar edema, generating copious, pink, frothy sputum [9]. In view of the above, NPPE induction can be considered to be noncardiogenic. It is generally regarded that greater the negative intrapleural pressure, higher the risk of pulmonary edema. This explains why NPPE occurs more often in healthy young men $[8,10]$.

In the present case, the point that the patient was young and in good physical health despite being a female, may have exacerbated NPPE on the same principle. In other words, it can be inferred that when neuromuscular recovery is made more rapidly and effectively during acute airway obstruction, paradoxically speaking, there is a higher possibility of NPPE owing to a sudden and sharp increase in negative intrapleural pressure. Such rapid and reliable reversal of neuromuscular blockade is feasible with sugammadex, a water-soluble, modified $\gamma$-cyclodextrin that rapidly encapsulates unbound rocuronium [11]. KhuenlBrady et al. [11] performed a randomized, actively controlled study which demonstrated that sugammadex provided faster mean time to recovery of the train-of-four (TOF) ratio to 0.9 in comparison to neostigmine (2.7 min versus $17.9 \mathrm{~min}$ ) after vecuronium-induced neuromuscular blockade. Similarly, sugammadex has been shown to rapidly counter the effects of rocuronium, usually within 1-2 minutes of administration [12].

It has been reported that residual neuromuscular blockade, defined as a normalized TOF ratio $<0.9$ after administration of reversal agent, after treatment with neostigmine was common in $63.5 \%$ of patients at tracheal extubation and in $56.5 \%$ on arrival at the PACU [13]. Furthermore, with every increase of 0.1 in the normalized TOF ratio at tracheal extubation the requirement of oxygen administration in the PACU was significantly lowered [13]. In the prospective, randomized study conducted by Berg et al. [14], it was observed that residual neuromuscular blockade is a risk factor for postoperative pulmonary complications such as pneumonia and atelectasis. In line with this, sugammadex effectively reduced the signs of residual postoperative neuromuscular blockade such as hypoxia, upper airway obstruction and decreased oxygen saturation, vis-à-vis conventional acetylcholinesterase inhibitors, which also the reason for preferred use of sugammadex.

Contrary to these innovative and beneficial outcomes in postoperative respiratory and pulmonary function, the occurrence of NPPE with the use of sugammadex during various conditions of acute airway obstruction warrants extreme vigilance as in our case. The patient in our case study was an otherwise healthy young woman who had undergone total thyroidectomy prone to laryngospasm [5]. During her first surgery, the patient developed post-extubation laryngospasm which may have got resolved with applying positive pressure ventilation with $100 \%$ $\mathrm{O}_{2}$ before the application of sugammadex. However, during her second operation, post-extubation laryngospasm occurred once again despite reversal of neuromuscular relaxation with sugammadex $(2 \mathrm{mg} / \mathrm{kg})$ and complete awakening of the patient. This indicated that sugammadex may dramatically trigger negative intrathoracic pressure by inducing a rapid and efficacious recovery of the respiratory muscle strength during acute upper airway obstruction. It could be speculated that in the first operation, NPPE did not occur as sugammadex was administered after induction of laryngospasm in comparison to the second operation in which NPPE happened as laryngospasm occurred even after reversal of neuromuscular blockade by sugammadex. The latter effect of sugammadex could be explained on the basis of a study by Suzuki et al. [2] wherein a possibility of induction of upper airway obstruction by increased upper airway collapsibility and large inspiratory forces applied by the diaphragm, that had fully recovered from muscle relaxation with the use of sugammadex, has been reported. Whether sugammadex has an effect on upper airway obstruction as a result of the difference in recovery profile between the upper airway muscle and diaghragm has not been investigated. Ikeda-Miyagawa et al. [15] have also reported a case of NPPE following laryngospasm immediately after administration of sugammadex under general anesthesia with laryngeal mask airway. Furthermore, the positive resuscitative fluid as in the second operation $(2,080 \mathrm{ml})$ may have contributed to the increase in pulmonary hydrostatic pressure gradient, favoring the transduction of fluid into the interstitial space $[11,15]$.

Therefore, during application of anesthesia, it is crucial to avoid the occurrence of upper airway obstruction, especially laryngospasm. The moment of tracheal extubation is highly critical in the prevention of laryngospasm which can be achieved by not stimulating the patient during extubation, removing the tube with the patient completely awake or extubating the tube in the deep plane [4].

In conclusion, patients at high risk of developing upper airway obstruction should be kept under close surveillance of an anesthesiologist due to the possibility of NPPE occurrence even when neuromuscular blockade is reversed by sugammadex, though it is beneficial under certain circumstances.

\section{References}

1. Olsson GL, Hallen B. Laryngospasm during anaesthesia. A computer-aided incidence study in 136,929 patients. Acta Anaesthesiol Scand 1984; $28: 567-75$. 
2. Suzuki M, Inagi T, Kikutani T, Mishima T, Bito H. Negative pressure pulmonary edema after reversing rocuronium-induced neuromuscular blockade by sugammadex. Case Rep Anesthesiol 2014; 2014: 135032.

3. Landsman IS. Mechanisms and treatment of laryngospasm. Int Anesthesiol Clin 1997; 35: 67-73.

4. Hobaika AB, Lorentz MN. Laryngospasm. Rev Bras Anestesiol 2009; 59: 487-95.

5. Ghofaily LA, Simmons C, Chen L, Liu R. Negative pressure pulmonary edema after laryngospasm: a revisit with a case report. J Anesth Clin Res 2013; 3: 252.

6. Guffin TN, Har-el G, Sanders A, Lucente FE, Nash M. Acute postobstructive pulmonary edema. Otolaryngol Head Neck Surg 1995; 112: 235-7.

7. Udeshi A, Cantie SM, Pierre E. Postobstructive pulmonary edema. J Crit Care 2010; 25: 508.e1-5.

8. Van Kooy MA, Gargiulo RF. Postobstructive pulmonary edema. Am Fam Physician 2000; 62: 401-4.

9. Gupta M, Akhavan A, Hall M, Palese M. Negative pressure pulmonary edema after laparoscopic donor nephrectomy. JSLS 2012; 16: 647-9.

10. Anderson AF, Alfrey D, Lipscomb AB Jr. Acute pulmonary edema, an unusual complication following arthroscopy: a report of three cases. Arthroscopy 1990; 6: 235-7.

11. Khuenl-Brady KS, Wattwil M, Vanacker BF, Lora-Tamayo JI, Rietbergen H, Alvarez-Gómez JA. Sugammadex provides faster reversal of vecuronium-induced neuromuscular blockade compared with neostigmine: a multicenter, randomized, controlled trial. Anesth Analg 2010; 110: $64-73$.

12. Sorgenfrei IF, Norrild K, Larsen PB, Stensballe J, Ostergaard D, Prins ME, et al. Reversal of rocuronium-induced neuromuscular block by the selective relaxant binding agent sugammadex: a dose-finding and safety study. Anesthesiology 2006; 104: 667-74.

13. Fortier LP, McKeen D, Turner K, de Médicis É, Warriner B, Jones PM, et al. The RECITE study: a canadian prospective, multicenter study of the incidence and severity of residual neuromuscular blockade. Anesth Analg 2015; 121: 366-72.

14. Berg H, Roed J, Viby-Mogensen J, Mortensen CR, Engbaek J, Skovgaard LT, et al. Residual neuromuscular block is a risk factor for postoperative pulmonary complications. A prospective, randomised, and blinded study of postoperative pulmonary complications after atracurium, vecuronium and pancuronium. Acta Anaesthesiol Scand 1997; 41: 1095-103.

15. Ikeda-Miyagawa Y, Kihara T, Matsuda R. Case of negative pressure pulmonary edema after administration of sugammadex under general anesthesia with laryngeal mask airway. Masui 2014; 63: 1362-5. 